\title{
高立体、区域选择性合成(Z)-1-芳硫基-2-芳硒基烯
}

\author{
谭 平 ${ }^{a, b}$ 李宁波 ${ }^{b}$ 许新华*,b \\ ( ${ }^{a}$ 湖南机电职业技术学院生物与化学工程系 长沙 410151) \\ ( ${ }^{b}$ 湖南大学化学化工学院 长沙 410082)
}

\begin{abstract}
摘要 氢氧化铯催化下，四氢呋喃作溶剂，室温、氮气保护下，炔基硫醚与硒酚发生亲核加成反应，立体选择性合成一 系列(Z)-1-芳硫基-2-芳硒基烯，收率 70\% 93\%. 反应机理为氢氧化铯与硒酚反应产生的芳硒化铯，随后对炔硫醚进行 亲核加成, 形成的烯基负离子水解得到产物.

关键词 亲核加成; 立体选择性性; 炔基硫醚; 氢氧化铯; (Z)-1-芳硫基-2-芳硒基烯
\end{abstract}

\section{Highly Regio- and Stereo-selective Synthesis of (Z)-1-Arylthio-2-Arylseleno Alkenes}

\author{
Tan, Ping ${ }^{a, b} \quad$ Li, Ningbo ${ }^{b} \quad$ Xu, Xinhua ${ }^{*, b}$ \\ ( ${ }^{a}$ Department of Biological and Chemical Engineering, Hunan Mechanical and Electrical Polytechnic, Changsha 410151) \\ ( ${ }^{b}$ College of Chemistry and Chemical Engineering, Hunan University, Changsha 410082)
}

\begin{abstract}
In the presence of catalytic amount of cesium hydroxide, the nucleophilic addition reaction of selenols to alkyne sulfurnides occurred in tetrahydrofuran at room temperature under nitrogen atmosphere to give highly regio- and stereo-selective (Z)-1-arylthio-2-arylseleno alkenes in 70\% 93\% yields. The mechanism involved the formation of cesium arylselenides and the nucleophilic addition to the alkynyl sulfides in the presence of cesium hydroxide. The corresponding alkenes were obtained upon hydrolysis.

Keywords nucleophilic addition; regioselectivity; alkynyl thioether; cesium hydroxide; (Z)-1-arylthio-2-arylseleno alkenes
\end{abstract}

双杂取代烯烃既是合成转化的重要中间体，又是络 合化学的双齿配体及微电子光学材料的前体. 不对称的 1,2-二杂取代烯烃, 即两个双键碳原子连接不同的杂原 子的烯烃，由于两个杂原子具有不同的性质和反应活 性, 因此, 这类杂取代烯烃比对应的对称的 1,2-二杂取 代烯烃在有机合成中具有更重要的作用. 关于对称二杂 取代烯烃的制备, 文献报道了很多方法, 主要通过过渡 金属 ${ }^{[1 \sim 15]}$ 或铯碱 ${ }^{[16 ~ 19]}$ 催化二杂醚与炔烃的加成; 对于 不对称的 1,2-二杂取代烯烃的制备方法的报道不 多 ${ }^{[20 ~ 23]}$. Dabdoub 等 ${ }^{[24]}$ 报道了炔基硫醚与二杂醚 $\mathrm{NaBH}_{4}$ 反应形成不对称的 1,2-二杂取代烯, 这一方法要用计量 的还原剂 $/ \mathrm{NaBH}_{4}$; Zeni 课题组 ${ }^{[20]}$ 报道了利用不对称二杂 醚(ArSSeAr)与炔烃反应合成不对称的 1,2-而杂取代烯, 但这方法要使用对空气高度敏感的丁基锂试剂, 而且, 这一方法适用范围很少，仅仅适用于 $\beta$ 位有羟基的炔烃，
即炔丙醇类. 本课题组利用氢氧化铯作催化剂, 成功的 实现了不对称二杂醚 $(\mathrm{ArSSeAr})$ 与炔烃的加成, 转化率 近 $100 \%$ ，反应具有专一的立体和区域选择性，且适用 于多种炔, 不需无水条件. 尽管, 本课题组的这一方法 有很大优势，但是不对称二杂醚(ArSSeAr)试剂不易获 得。炔硒醚及炔硫醚是重要合成中间体，且易于制

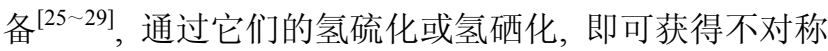
的 1,2-二杂取代烯烃. 本文报道氢氧化铯催化炔硫醚氢 硒化反应，高立体、区域选择性合成(Z)-1-芳硫基-2-芳硒 基烯.

\section{1 实验部分}

\section{1 仪器与试剂}

${ }^{1} \mathrm{H}$ NMR (以 TMS 为内标), ${ }^{13} \mathrm{C}$ NMR(以 TMS 为内 标)用 INOVA-400 型仪测定; 质谱由 HP5989A 测定; 元

*E-mail: xhx1581@yahoo.com.cn

Received June 10, 2012; revised July 13, 2012; published online July 19, 2012.

Project supported by the National Natural Science Foundation of China (No. 21172061).

国家自然科学基金(No. 21172061)资助项目. 
素分析用 Yanaco CHN CORDER MT-3 型自动元素分析 仪测定. 溶剂四氢呋喃未经处理, 硅胶为青岛海洋化工 厂产品, 氢氧化铯从 Aldrich 公司购买.

\section{2 实验方法}

将 THF $3.0 \mathrm{~mL}$, 炔硫醚 $1.0 \mathrm{mmol}$, 硒酚 $1.1 \mathrm{mmol}$ 及氢氧化铯 $0.2 \mathrm{mmol}$ 加入到圆底烧瓶中, 在室温、氮气 的保护下搅拌. TLC 跟踪反应, 炔硫醚完全反应. 减压 下, 旋干溶剂 THF, 用石油醚 $50 \mathrm{~mL}$ 稀释, 加入 $10 \mathrm{~mL}$ 水, 将有机相旋蒸去掉溶剂, 剩余物通过柱层析纯化, 用石油醚/乙醚 $(V / V=30 / 1)$ 作洗脱剂, 得目标产物 $\mathbf{3 a} \sim$ 31.

(Z)-1-苯硫基-2-苯硒基-3-羟基-1-丙烯(3a): 浅黄色 油状液体. ${ }^{1} \mathrm{H}$ NMR $\left(\mathrm{CDCl}_{3}, 400 \mathrm{MHz}\right) \delta: 7.46 \sim 7.51(\mathrm{~m}$, 2H), $7.21 \sim 7.38(\mathrm{~m}, 9 \mathrm{H}), 4.14(\mathrm{~s}, 2 \mathrm{H}), 2.30(\mathrm{~s}, 1 \mathrm{H}) ;{ }^{13} \mathrm{C}$ NMR $\left(\mathrm{CDCl}_{3}, 100 \mathrm{MHz}\right) \delta$ : 134.43, 133.65, 132.95, 132.04, 130.83, 129.63, 129.34, 129.15, 127.83, 126.54, 65.72; MS (EI, $70 \mathrm{eV}) \mathrm{m} / z$ : $320\left(\mathrm{M}^{+}\right)$. Anal. calcd for $\mathrm{C}_{15} \mathrm{H}_{14} \mathrm{OSSe}$ : C 56.07, H 4.39; found C 56.28, H 4.53.

(Z)-1-苯硫基-2-苯硒基-3-甲氧基-1-丙烯 (3b): 浅黄 色油状液体. ${ }^{1} \mathrm{H}$ NMR $\left(\mathrm{CDCl}_{3}, 400 \mathrm{MHz}\right) \delta: 7.13 \sim 7.55$ $(\mathrm{m}, 11 \mathrm{H}), 3.84(\mathrm{~s}, 2 \mathrm{H}), 3.19(\mathrm{~s}, 3 \mathrm{H}) ;{ }^{13} \mathrm{C}$ NMR $\left(\mathrm{CDCl}_{3}\right.$, $100 \mathrm{MHz}) \delta$ : 133.66, 133.23, 133.10, 129.89, 129.33, 129.02, 127.76, 126.88, 74.65, 57.45; MS (EI, $70 \mathrm{eV}) \mathrm{m} / \mathrm{z}$ : $336\left(\mathrm{M}^{+}\right)$. Anal. calcd for $\mathrm{C}_{16} \mathrm{H}_{16}$ OSSe: C 57.36, H 4.89; found C 57.42, H 4.82 .

(Z)-1-苯硫基-2-对甲苯硒基-3-甲氧基-1-丙烯(3c): 浅黄色油状液体. ${ }^{1} \mathrm{H}$ NMR $\left(\mathrm{CDCl}_{3}, 400 \mathrm{MHz}\right) \delta: 7.21 \sim$ $7.64(\mathrm{~m}, 10 \mathrm{H}), 3.96$ (s, 2H), 3.35 (s, 3H), $2.31(\mathrm{~s}, 3 \mathrm{H}) ;{ }^{13} \mathrm{C}$ NMR $\left(\mathrm{CDCl}_{3}, 100 \mathrm{MHz}\right) \delta$ : $133.72,133.32,133.16$, $129.94,129.35,129.12,127.80,126.95,74.74,58.64$, 21.56; MS (EI, $70 \mathrm{eV}) \mathrm{m} / z$ : $350\left(\mathrm{M}^{+}\right)$. Anal. calcd for $\mathrm{C}_{17} \mathrm{H}_{18} \mathrm{OSSe}$ : C 58.38, H 5.16; found C 58.41, H 4.20.

(Z)-1-苯硫基-2-邻溴苯硒基-3-甲氧基-1-丙烯(3d): 黄色油状液体. ${ }^{1} \mathrm{H} \mathrm{NMR}\left(\mathrm{CDCl}_{3}, 400 \mathrm{MHz}\right) \delta: 7.18 \sim 7.52$ (m, 9H), 6.97 (s, 1H), 3.90 (s, 2H), 3.31 (s, 3H); ${ }^{13} \mathrm{C}$ NMR $\left(\mathrm{CDCl}_{3}, 100 \mathrm{MHz}\right) \delta: 136.61,135.02,134.22,133.42$, 129.96, 129.31, 128.63, 128.01, 127.74, 127.25, 123.51, 75.24, 58.36; MS (EI, $70 \mathrm{eV}) \mathrm{m} / \mathrm{z}$ : $414\left(\mathrm{M}^{+}+1\right)$. Anal. calcd for $\mathrm{C}_{16} \mathrm{H}_{15}$ BrOSSe: C 46.38, H 3.62; found C 46.34, H 3.59 .

(Z)-1-苯硫基-2-苯硒基-3-羊基-3-甲基-1-丁烯(3e): 浅黄色油状液体. ${ }^{1} \mathrm{H}$ NMR $\left(\mathrm{CDCl}_{3}, 400 \mathrm{MHz}\right) \delta: 7.60(\mathrm{~s}$, $1 \mathrm{H}), 7.47 \sim 7.50(\mathrm{~m}, 2 \mathrm{H}), 7.24 \sim 7.37(\mathrm{~m}, 8 \mathrm{H}), 2.35(\mathrm{~s}$, 1H), 1.38 (s, 6H); ${ }^{13} \mathrm{C}$ NMR $\left(\mathrm{CDCl}_{3}, 100 \mathrm{MHz}\right) \delta: 138.24$, $137.15,135.24,133.53,129.83,129.15,128.68,127.74$,
127.02, 125.45, 75.47, 29.16; MS (EI, $70 \mathrm{eV}$ ) m/z: 349 $\left(\mathrm{M}^{+}\right)$. Anal. calcd for $\mathrm{C}_{17} \mathrm{H}_{18}$ OSSe: C 58.45, H 5.16; found C 58.50, H 5.19.

(Z)-1-苯硫基-2-间氯苯硒基-3-羟基-3-二甲基-1-丙 烯(3f): 浅黄色油状液体. ${ }^{1} \mathrm{H} \mathrm{NMR}\left(\mathrm{CDCl}_{3}, 400 \mathrm{MHz}\right) \delta$ : $7.58(\mathrm{~s}, 1 \mathrm{H}), 7.47 \sim 7.51(\mathrm{~m}, 2 \mathrm{H}), 7.11 \sim 7.32(\mathrm{~m}, 7 \mathrm{H})$, $2.28(\mathrm{~s}, 1 \mathrm{H}), 1.35(\mathrm{~s}, 6 \mathrm{H}) ;{ }^{13} \mathrm{C} \mathrm{NMR}\left(\mathrm{CDCl}_{3}, 100 \mathrm{MHz}\right) \delta$ : 138.30, 137.19, 136.63, 134.89, 133.31, 129.97, 129.53, 127.78, 126.43, 125.67, 124.85, 75.45, 29.32; MS (EI, 70 eV) $m / z: 382\left(\mathrm{M}^{+}\right)$. Anal. calcd for $\mathrm{C}_{17} \mathrm{H}_{17} \mathrm{ClOSSe}$ C 53.20, H 4.46; found C 53.27, H 4.52.

(Z)-1-苯硫基-2-苯硒基-3-羟基-3-甲基-3-乙基-1-丙 烯(3g): 浅黄色油状液体. ${ }^{1} \mathrm{H} \mathrm{NMR}\left(\mathrm{CDCl}_{3}, 400 \mathrm{MHz}\right) \delta$ : $7.42 \sim 7.51(\mathrm{~m}, 3 \mathrm{H}), 7.08 \sim 7.24(\mathrm{~m}, 8 \mathrm{H}), 2.19(\mathrm{~s}, 1 \mathrm{H})$, 1.54 (q, $J=7.4 \mathrm{~Hz}, 2 \mathrm{H}), 1.28$ (s, 3H), 0.91 (t, $J=7.4 \mathrm{~Hz}$, $3 \mathrm{H}) ;{ }^{13} \mathrm{C} \mathrm{NMR}\left(\mathrm{CDCl}_{3}, 100 \mathrm{MHz}\right) \delta: 138.24,137.41$, 135.21, 133.14, 131.21, 129.41, 128.85, 127.32, 127.10, 125.48, 78.34, 33.61, 26.43, 23.35; MS (EI, $70 \mathrm{eV}$ ) $\mathrm{m} / \mathrm{z}$ : $363\left(\mathrm{M}^{+}\right)$. Anal. calcd for $\mathrm{C}_{18} \mathrm{H}_{20} \mathrm{OSSe}$. C 59.51, H 5.54; found C 59.57, H5.59.

(Z)-1-苯硫基-2-苯硒基-3-羟基-3-环已基-1-丙烯 (3h): 浅黄色油状液体. ${ }^{1} \mathrm{H}$ NMR $\left(\mathrm{CDCl}_{3}, 400 \mathrm{MHz}\right) \delta$ : $7.55(\mathrm{~s}, 1 \mathrm{H}), 7.48 \sim 7.53(\mathrm{~m}, 2 \mathrm{H}), 7.12 \sim 7.30(\mathrm{~m}, 8 \mathrm{H})$, $1.78(\mathrm{~s}, 1 \mathrm{H}), 1.52 \sim 1.68(\mathrm{~m}, 10 \mathrm{H}) ;{ }^{13} \mathrm{C} \mathrm{NMR}\left(\mathrm{CDCl}_{3}, 100\right.$ MHz) $\delta: 138.67,137.72,135.47,134.23,131.12,129.53$, 128.90, 127.43, 126.97, 125.48, 71.61, 36.14, 25.24, 21.80; MS (EI, $70 \mathrm{eV}) \mathrm{m} / z$ : $389\left(\mathrm{M}^{+}\right)$. Anal. calcd for $\mathrm{C}_{20} \mathrm{H}_{22} \mathrm{OSSe}$ : C 61.70, H 5.66; found C 61.63, H 5.60.

(Z)-1-苯硫基-2-苯硒基-3-羟基-3-乙基-1-丙烯(3i): 浅黄色油状液体. ${ }^{1} \mathrm{H}$ NMR $\left(\mathrm{CDCl}_{3}, 400 \mathrm{MHz}\right) \delta: 7.52 \sim$ $7.57(\mathrm{~m}, 2 \mathrm{H}), 7.31 \sim 7.35(\mathrm{~m}, 3 \mathrm{H}), 7.18 \sim 7.24(\mathrm{~m}, 5 \mathrm{H})$, $4.16(\mathrm{t}, J=7.4 \mathrm{~Hz}, 1 \mathrm{H}), 2.38(\mathrm{~s}, 1 \mathrm{H}), 1.43 \sim 1.62(\mathrm{~m}, 2 \mathrm{H})$, $0.92(\mathrm{t}, J=7.4 \mathrm{~Hz}, 3 \mathrm{H}) ;{ }^{13} \mathrm{C} \mathrm{NMR}\left(\mathrm{CDCl}_{3}, 100 \mathrm{MHz}\right) \delta$ : 136.18, 135.09, 133.78, 132.89, 130.18, 129.16, 128.96, 128.61, 127.59, 126.30, 76.71, 28.88, 9.69; MS (EI, $70 \mathrm{eV}$ ) $m / z: 349\left(\mathrm{M}^{+}\right)$. Anal. calcd for $\mathrm{C}_{17} \mathrm{H}_{18} \mathrm{OSSe}$ : C 58.45, $\mathrm{H}$ 5.19; found C 58.29, H 4.91 .

(Z)-1-苯硫基-2-苯硒基-3-苯氧基-1-丙烯 $(3 \mathbf{j})$ : 黄色 油状液体. ${ }^{1} \mathrm{H}$ NMR $\left(\mathrm{CDCl}_{3}, 400 \mathrm{MHz}\right) \delta: 7.51 \sim 7.57$ (m, $2 \mathrm{H}), 7.48 \sim 7.50(\mathrm{~m}, 2 \mathrm{H}), 7.43(\mathrm{~s}, 1 \mathrm{H}), 7.27 \sim 7.34(\mathrm{~m}$, $6 \mathrm{H}), 7.16(\mathrm{t}, J=8.0 \mathrm{~Hz}, 2 \mathrm{H}), 6.86(\mathrm{t}, J=7.2 \mathrm{~Hz}, 1 \mathrm{H}), 6.54$ (d, $J=8.0 \mathrm{~Hz}, 2 \mathrm{H}), 4.48$ (s, 2H); ${ }^{13} \mathrm{C}$ NMR $\left(\mathrm{CDCl}_{3}, 100\right.$ MHz) $\delta: 157.32,135.16,133.24,132.76,130.41,129.31$, $129.12,128.89,128.45,128.10,127.79,127.61,122.41$, 115.13, 72.44; MS (EI, $70 \mathrm{eV}) \mathrm{m} / z$ : $398\left(\mathrm{M}^{+}\right)$. Anal. calcd 
for $\mathrm{C}_{21} \mathrm{H}_{18} \mathrm{OSSe}$ : C 63.32, H 4.52; found C 63.38, H 4.57.

(Z)-1-苯硫基-2-对甲苯硒基-3-苯氧基-1-丙烯(3k): 浅黄色油状液体. ${ }^{1} \mathrm{H} \mathrm{NMR}\left(\mathrm{CDCl}_{3}, 400 \mathrm{MHz}\right) \delta: 7.50$ (d, $J=7.0 \mathrm{~Hz}, 2 \mathrm{H}), 7.41(\mathrm{~m}, 3 \mathrm{H}), 7.18 \sim 7.22(\mathrm{~m}, 2 \mathrm{H}), 7.08$ $(\mathrm{d}, J=8.0 \mathrm{~Hz}, 4 \mathrm{H}), 6.84$ (t, $J=6.8 \mathrm{~Hz}, 1 \mathrm{H}), 6.64$ (d, $J=8.0$ $\mathrm{Hz}, 2 \mathrm{H}), 4.42$ (s, 2H), 2.30 (s, 3H); ${ }^{13} \mathrm{C} \mathrm{NMR}\left(\mathrm{CDCl}_{3}, 100\right.$ $\mathrm{MHz}) \delta: 158.71,139.32,138.64,133.87,133.41,133.12$, 130.42 , 130.18, 129.91, 127.48, 127.21, 125.26, 121.40, 117.18, 73.56, 23.41; MS (EI, $70 \mathrm{eV}) \mathrm{m} / z: 412\left(\mathrm{M}^{+}\right)$. Anal. calcd for $\mathrm{C}_{22} \mathrm{H}_{20} \mathrm{OSSe}$ : C 64.08, H 4.85; found C 64.13, H 4.89 .

(Z)-1-苯硫基-2-苯硒基乙烯(3I): 黄色油状液体. ${ }^{1} \mathrm{H}$ NMR $\left(\mathrm{CDCl}_{3}, 400 \mathrm{MHz}\right) \delta: 7.40 \sim 7.51(\mathrm{~m}, 6 \mathrm{H}), 7.20 \sim$ $7.31(\mathrm{~m}, 9 \mathrm{H}), 7.01(\mathrm{~s}, 1 \mathrm{H}) ;{ }^{13} \mathrm{C} \mathrm{NMR}\left(\mathrm{CDCl}_{3}, 100 \mathrm{MHz}\right) \delta$ : $133.76,133.32,132.56,132.21,129.89,129.64,129.23$, 128.94, 128.73, 128.62, 128.20, 127.46, 127.21, 126.32; MS (EI, $70 \mathrm{eV}) \mathrm{m} / z$ : $368\left(\mathrm{M}^{+}\right)$. Anal. calcd for $\mathrm{C}_{20} \mathrm{H}_{16}$ OSSe: C 65.22, H 4.35; found C 65.27, H 4.41.

\section{2 结果与讨论}

\section{1 反应条件探索}

首先，以 3-甲氧基丙炔基苯基硫醚与苯硒酚加成以 模型(Eq. 1), 探讨溶剂对反应的影响. 结果见表 1 .

$$
\left.\mathrm{H}_{3} \mathrm{COH}_{2} \mathrm{C}=\mathrm{SPh}+\mathrm{PhSeH} \stackrel{\mathrm{CsOH} 20 \mathrm{~mol}_{2} \mathrm{H}_{3} \mathrm{COH}_{2} \mathrm{C}}{\longrightarrow}\right\rangle_{\mathrm{PhSe}}^{\mathrm{H}}(1)
$$

表 1 溶剂对反应影响

Table 1 Influence of solvents on the reaction

\begin{tabular}{clcccc}
\hline Entry & Solvent & $T /{ }^{\circ} \mathrm{C}$ & Time/h & Yield & $Z / E$ \\
\hline 1 & $\mathrm{CH}_{3} \mathrm{OH}$ & 30 & 3.0 & 36 & $100: 0$ \\
2 & $\mathrm{CH}_{3} \mathrm{CH}_{2} \mathrm{OH}$ & 30 & 3.0 & 38 & $100: 0$ \\
3 & $\mathrm{THF}$ & 30 & 3.0 & 92 & $100: 0$ \\
4 & $\mathrm{DMF}$ & 30 & 3.0 & 95 & $100: 0$ \\
5 & $\mathrm{DMSO}$ & 30 & 3.0 & 93 & $100: 0$ \\
6 & $\mathrm{CH}_{3} \mathrm{CH}_{2} \mathrm{OH}$ & 30 & 10 & 23 & $100: 0$ \\
7 & $\mathrm{CH}_{3} \mathrm{CH}_{2} \mathrm{OH}$ & 60 & 3.0 & 86 & $85: 15$ \\
\hline
\end{tabular}

由表 1 可知, 在 $30{ }^{\circ} \mathrm{C}$ 时, 氢氧化铯 $(0.2 \mathrm{mmol})$ 存在 下，3-甲氧基丙炔基苯基硫醚(1.0 mmol)与苯硒酚(1.1 $\mathrm{mmol}$ ) 在 THF, DMF 及 DMSO 中反应 $3.0 \mathrm{~h}$, 高产率、高 立体、区域选择性的形成 1-苯硫基-2-苯硒基-3-甲氧基 丙烯; 以甲醇或乙醇作溶剂, 在 $30{ }^{\circ} \mathrm{C}$ 转化率较低, 在 $60{ }^{\circ} \mathrm{C}$, 产率迅速提高, 但形成 $15 \%$ 的 $E$ 异构体. 由于 THF 沸点较低, 后处理简单, 所以, THF 是最佳溶剂.

\section{2 催化剂对比}

以 3-甲氧基丙炔基苯基硫醚与苯硒酚加成为模型, 探讨碱金属氢氧化物对反应的影响，结果见表 2 .
表 2 不同碱金属氢氧化物催化下对反应产率影响

Table 2 Influence of various alkali metal hydroxides on the reaction yields

\begin{tabular}{lcccccc}
\hline \multicolumn{1}{c}{ Entry } & 1 & 2 & 3 & 4 & 5 & 6 \\
\hline Base & $\mathrm{LiOH}$ & $\mathrm{NaOH}$ & $\mathrm{KOH}$ & $\mathrm{RbOH}$ & $\mathrm{CsOH}$ & $\mathrm{Cs}_{2} \mathrm{CO}_{3}$ \\
Yield $^{a} \%$ & 5.6 & 43 & 58 & 73 & 93 & 82 \\
\hline
\end{tabular}

由表 2 可知, 在相同条件下, $\mathrm{CsOH}$ 表现出最好的 催化效果. 其原理是由于在上述碱金属氢氧化物中, 铯 离子具有最大的体积, 与氢氧根离子间静电引力最小, 导致 $\mathrm{CsOH}$ 中 $\mathrm{OH}^{-}$表现出强的碱性, $\mathrm{CsOH}$ 与硒酚反应 形成的芳硒化铯 $\mathrm{ArSeCs}$ 中芳硒负离子 $\mathrm{ArSe}^{-}$表现出强 的亲核性.

\section{3 反应底物拓展}

在此条件下，考察了各种炔基硫醚与不同硒酚的加 成，结果见表 3 .

表 3 (Z)-1-芳硫基-2-芳硒基烯的合成

Table 3 Synthesis of (Z)-1-arylthio-2-arylselebno-1-alkene

$$
\mathrm{R}=\mathrm{SPh}+\mathrm{ArSeH} \stackrel{\mathrm{CsOH}(20 \mathrm{~mol} \%)}{\mathrm{THF}, \text { r.t. }} \underset{\substack{\mathrm{ArSe} \\ \mathbf{3 a} \sim \mathbf{3 l}}}{\mathrm{R}} \sum_{\mathrm{SPh}}^{\mathrm{R}}
$$

\begin{tabular}{cllc}
\hline Compd. & \multicolumn{1}{c}{$\mathrm{R}$} & \multicolumn{1}{c}{$\mathrm{Ar}$} & Yield/\% \\
\hline 3a & $\mathrm{HOCH}_{2}$ & $\mathrm{C}_{6} \mathrm{H}_{5}$ & 82 \\
3b & $\mathrm{CH}_{3} \mathrm{OCH}_{2}$ & $\mathrm{C}_{6} \mathrm{H}_{5}$ & 93 \\
3c & $\mathrm{CH}_{3} \mathrm{OCH}_{2}$ & $p-\mathrm{CH}_{3} \mathrm{C}_{6} \mathrm{H}_{4}$ & 90 \\
3d & $\mathrm{CH}_{3} \mathrm{OCH}_{2}$ & $o-\mathrm{BrC}_{6} \mathrm{H}_{4}$ & 87 \\
3e & $\mathrm{HO}\left(\mathrm{CH}_{3}\right)_{2} \mathrm{C}$ & $\mathrm{C}_{6} \mathrm{H}_{5}$ & 70 \\
3f & $\mathrm{HO}\left(\mathrm{CH}_{3}\right)_{2} \mathrm{C}$ & $m-\mathrm{ClC}_{6} \mathrm{H}_{4}$ & 75 \\
3g & $\mathrm{HO}\left(\mathrm{CH}_{3}\right)\left(\mathrm{C}_{2} \mathrm{H}_{5}\right) \mathrm{C}$ & $\mathrm{C}_{6} \mathrm{H}_{5}$ & 80 \\
3h & $\mathrm{HO}\left(\mathrm{C}_{6} \mathrm{H}_{11}\right) \mathrm{CH}$ & $\mathrm{C}_{6} \mathrm{H}_{5}$ & 86 \\
3i & $\mathrm{HO}\left(\mathrm{C}_{2} \mathrm{H}_{5}\right) \mathrm{CH}$ & $\mathrm{C}_{6} \mathrm{H}_{5}$ & 95 \\
3j & $\mathrm{PhOCH}$ & $\mathrm{Ph}_{2}$ & 85 \\
3k & $\mathrm{PhOCH}$ & $p-\mathrm{CH}_{3} \mathrm{C}_{6} \mathrm{H}_{4}$ & 83 \\
3I & $\mathrm{Ph}$ & $\mathrm{Ph}$ & 84 \\
3h & $n-\mathrm{C}_{4} \mathrm{H}_{9}$ & $\mathrm{Ph}$ & $\mathrm{NR}$ \\
\hline
\end{tabular}

表 3 结果表明，以 THF 作溶剂，在 $20 \mathrm{mmol} \% \mathrm{CsOH}$ 存在下，3-羊基丙炔基硫醚、3-甲氧基丙炔基硫醚、3苯氧基丙炔基硫醚、苯乙炔基硫醚与各种硒酚在室温、 氮气氛中都能有效进行亲核加成，高立体和区域选择性 的形成(Z)-1-芳硫基-2-芳硒基烯. 苯环上取代基的性质 对反应影响不大，不论是吸电子基还是给电子基，加成 反应都能很好进行. 但是, 炔基上取代基的性质，对反 应有显著的影响，烷基炔基硫醚与苯硒酚不发生反应.

产物结构通过 ${ }^{1} \mathrm{H} N M R,{ }^{13} \mathrm{C} \mathrm{NMR}, \mathrm{MS}, \mathrm{NOE}$ 和 NOESY 确证. 化合物 $\mathbf{3 a} \sim \mathbf{3 d}, \mathbf{3 h} \sim \mathbf{3 k}$ 的 ${ }^{1} \mathrm{H}$ NMR 中, 双 键 $\mathrm{H}$ 与亚甲基 $\mathrm{CH}_{2}$ 或次甲基 $\mathrm{CH}$ 中 $\mathrm{H}$ 偶合常数为 $1.6 \mathrm{~Hz}$ 左右，是弱偶合，表明双键 $\mathrm{H}$ 与亚甲基 $\mathrm{CH}_{2}$ 或次甲基 $\mathrm{CH}$ 不是连接在同一双键碳原子上，因此它们为 1-芳硫 
基-2-芳硒基-1-烯; 如果双键 $\mathrm{H}$ 与亚甲基 $\mathrm{CH}_{2}$ 或次甲基 $\mathrm{CH}$ 连接在同一双键碳原子上, 那么, 它们之间会产生 强的偶合, 其偶合常数一般 $\delta 6.0$ 左右. 产物的立体结构 分别通过 NOE 和 NOESY 实验确证. 如化合物 $\mathbf{3 b}$ 中 $\mathrm{CH}_{2}$ 与双键上 $\mathrm{H}$ 有 $\mathrm{NOE}$ 效应，当用干扰场干扰双键上 $\mathrm{H}$, $\mathrm{CH}_{2}$ 积分有 $12 \%$ 的增强, 表明为 $Z$ 式构型. 如果是 $E$ 式 结构, $\mathrm{CH}_{2}$ 与双键上 $\mathrm{H}$ 就不会有 $\mathrm{NOE}$ 效应. 同样通过化 合物的 NOESY NMR 可以确证其立体结构表明. 化合 物 3b 与 3c 的 NOESY NMR 谱图上烯基氢与亚甲基氢 有交点，证明为 $Z$ 式构型.

\section{4 催化机理分析}

上述反应可能的反应机理如 Scheme 1 所示.

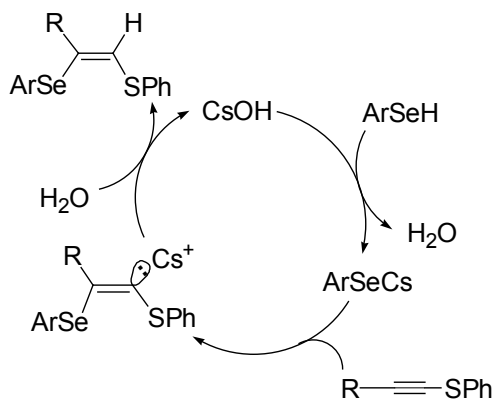

Scheme 1

在催化循环中, 氢氧化铯与硒酚反应产生的芳硒化 铯, 随后对炔硫醚进行亲核加成, 形成的烯基负离子水 解得到产物, 同时形成氢氧化铯. 芳硒化铯对炔硫醚进 行亲核加成具有选择性, 芳硒化铯进攻炔硫醚中与烷基 相连的炔基碳, 而不是进攻与芳硫基相连的炔基碳, 因 为前者形成的乙烯基碳负离子被硫原子分享而稳定.

\section{3 结论}

本方法具有反应条件温和, 高收率、高选择性等优 点，为合成(Z)-1-芳硫基-2-芳硒基烯提供了一条有效路 径.

\section{References}

[1] Kuniyasu, H.; Ogawa, A.; Miyazaki, S. I.; Ryu, I.; Kambe, N.; Sonoda, N. J. Am. Chem. Soc. 1991, 113, 9796.

[2] Moro, A. V.; Nogueira, C. W.; Barbosa, N. B. V. J. Org. Chem. 2005, 70, 5257.

[3] Ogawa, A.; Ikeda, T.; Kimura, K.; Hirao, T. J. Am. Chem. Soc. 1999, 121, 5108 .

[4] Ananikov, V. P.; Beletskaya, I. P.; Aleksandrov, G. G.; Eremenko,
I. L. Organometallics 2003, 22, 1414.

[5] Ogawa, A.; Kuniyasu, H.; Sonoda, N.; Hirao, T. J. Org. Chem. 1997, 62, 8361.

[6] Cai, M. Z.; Wang, Y. G.; Hao, W. Y. Green Chem. 2007, 9, 1180.

[7] Li, J. Y.; Liu, J.; Cai, M. Z. J. Chem. Res. 2009, 10, 616.

[8] Ananikov, V. P.; Kabeshov, M. A.; Beletskaya, I. P. J. Organomet. Chem. 2003, 687, 451.

[9] Ananikov, V. P.; Kabeshov, M. A.; Beletskaya, I. P. Synlett 2005, 1015.

[10] Ananikov, V. P.; Beletskaya, I. P. Org. Biomol. Chem. 2004, 2, 284.

[11] Ananikov, V. P.; Kabeshov, M. A.; Beletskaya, I. P. Organometallics 2005, 24, 1275.

[12] Usugi, S. I.; Yorimitsu, H.; Shinokubo, H.; Oshima, K. Org. Lett. 2004, 6, 601 .

[13] Dabdoub, M. J.; Jr. Guerrero, P. J. Tetrahedron Lett. 2001, 42, 7167.

[14] Watanabe, S. I.; Mori, E.; Nagai, H.; Iwamura, T.; Iwama, T.; Kataoka, T. J. Org. Chem. 2000, 65, 8893.

[15] Huang, X.; Xu, X. H.; Zheng, W. X. Synth. Commun. 1999, 29, 2399.

[16] Zeng, J. C.; Lu, R. L.; Xu, X. H.; Fang, J. K.; Zhou, B.; Zhang, Q. L. Chin. J. Org. Chem. 2004, 24, 1482 (in Chinese). (曾纪朝, 卢锐亮, 许新华, 方敬坤, 周冰, 张秋林, 有机化学, 2004, 24, 1482.)

[17] Zou, K. B.; Qiu, R. H.; Fang, D. W.; Liu, X. Y.; Xu, X. H. Synth. Commun. 2008, 38, 2237.

[18] Zou, K. B.; Yin, X. H.; Liu, W. Q.; Qiu, R. H.; Li, L. X.; Shao, L. L.; Li, Y. H.; Xu, X. H.; Yang, R. H. Synth. Commun. 2009, 39. 2646.

[19] Li, X. W.; Liu, W. Q.; Zou, K. B.; Xu, X. H. Chin. J. Org. Chem. 2007, 27, 1176 (in Chinese). (黎小武, 刘文奇, 邹康兵, 许新华, 有机化学, 2007, 27, 1176.)

[20] Schneider, C. C.; Godoi, B.; Prigol, M.; Nogueira, C. W.; Zeni, G. Organometallics 2007, 26, 4252.

[21] Perin, G.; Borges, E. L.; Alves, D. Tetrahedron Lett. 2012, 53, 2066.

[22] Ogawa, A.; Obayashi, R.; Ine, H.; Tsuboi, Y.; Sonoda, N.; Hirao, T. J. Org. Chem. 1998, 63, 881.

[23] Manarin, F.; Roehrs, J. A.; Prigol, M.; Alves, A.; Nogueira, C. W.; Zeni, G. Tetrahedron Lett. 2007, 48, 4805.

[24] Dabdoub, M. J.; Dabdoub, V. B.; Pereira, M. A. Tetrahedron Lett. 2001, 42, 1595.

[25] Tan, P.; Yin, X. H.; Yu, A. H.; Xu, X. H. Chin. J. Chem. 2011, 29, 765 (in Chinese) (谭平, 尹显洪, 喻爱和, 许新华, 中国化学, 2011, 29, 765.)

[26] Cohen, R. J.; Fox, D. L.; Salvatore, R. N. J. Org. Chem. 2004, 69, 4265.

[27] Lothar, W. B.; Margarete, F. S.; Paulo, H. M. Tetrahedron Lett. 2004, 45(13), 2735.

[28] Guerrero, P. G.; Dabdoub, M. J.; Marques, F. A.; Wosch, C. L.; Baroni, A. C. M.; Ferreira, A. G. Synth. Commun. 2008, 38, 4379.

[29] Xia, X.; Zou, K. B.; Fang, D. W.; Xu, X. H. Chin. J. Org. Chem. 2008, 28, 1487 (in Chinese).

(夏湘, 邹康兵, 方大为, 许新华, 有机化学, 2008, 28, 1487.)

(Li, L.; Lu, Z.) 